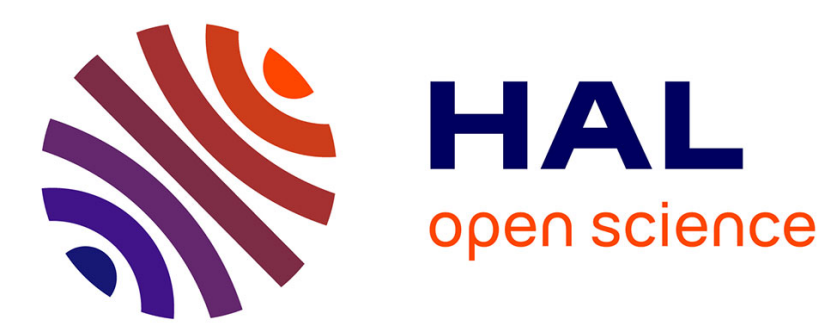

\title{
DIFFERENTIAL FORMS ON STRATIFIED SPACES II
}

Serap Gürer, Patrick Iglesias-Zemmour

\section{To cite this version:}

Serap Gürer, Patrick Iglesias-Zemmour. DIFFERENTIAL FORMS ON STRATIFIED SPACES II. Bulletin of the Australian Mathematical Society, 2018. hal-02401460

\section{HAL Id: hal-02401460 \\ https://hal.science/hal-02401460}

Submitted on 10 Dec 2019

HAL is a multi-disciplinary open access archive for the deposit and dissemination of scientific research documents, whether they are published or not. The documents may come from teaching and research institutions in France or abroad, or from public or private research centers.
L'archive ouverte pluridisciplinaire HAL, est destinée au dépôt et à la diffusion de documents scientifiques de niveau recherche, publiés ou non, émanant des établissements d'enseignement et de recherche français ou étrangers, des laboratoires publics ou privés. 


\title{
DIFFERENTIAL FORMS ON STRATIFIED SPACES II
}

\author{
SERAP GÜRER AND PATRICK IGLESIAS-ZEMMOUR
}

\begin{abstract}
AвSTRACT. We prove that, for a conelike stratified diffeological spaces, a zeroperverse form is the restriction of a global differential form if and only if its index is equal to 1 for every stratum.
\end{abstract}

\section{INTRODUCTION}

In the previous paper "Differential Forms On Stratified Spaces" [GIZ18], for each zero-perverse form defined on the regular part of a diffeological stratified space, we introduced an index for every stratum, counting the number of different forms generated by the zero-perverse form around the stratum. We showed that under some natural condition on the stratification - if two points could be connected by a path cutting the singular subset in a finite number of points - the zero-perverse form is the restriction of a differential form, defined globally on the space, if and only if the index is equal to 1 for every stratum.

In this paper, we prove this statement for locally conelike diffeological stratified spaces, without accessory conditions.

Thanks. - We are thankful to the referee who, by his relevant remarks, allowed us to improve the readability and the content of the paper.

\section{Locally Conelike Diffeological Stratified Spaces}

We suggest Benoit Kloeckner's survey [Klo07] for the classical topological approach of stratified spaces. About diffeology, we refer to the textbook [PIZ13], and about conelike stratified spaces, Larry Siebenmann's paper [Sie72].

Date: December 22, 2018.

1991 Mathematics Subject Classification. 58A35, 58A10.

Key words and phrases. Stratification, Diffeology, Differential Forms.

This research is partially supported by Tübitak, Career Grant No. 115F410, and the French-Turkish Research Fellowships Program, Embassy of France in Turkey 2017. The authors thank the «Espace Forbin» of the Faculty of Economy from Aix-Marseille University, and the people there, for their constant hospitality. 
Let us begin by specifying what we understand by Stratified Diffeological Spaces. Note that the topology of a diffeological space we referring to in the following is its natural D-Topology, defined in [PIZ13, \$2.8].

1. Stratified Diffeological Spaces. A stratification of a diffeological space $\mathrm{X}$ by manifolds is a finite family of subspaces $\mathscr{S}=\left\{S_{i}\right\}_{i \in \mathscr{I}}$, called strata, such that:

(1) The strata form a partition of $X$.

(2) Each stratum is a manifold for the subset diffeology ${ }^{1}$.

(3) The strata satisfy the frontier condition:

$$
\mathrm{S}_{i} \cap \overline{\mathrm{S}_{j}} \neq \varnothing \quad \Rightarrow \quad \mathrm{S}_{i} \subset \overline{\mathrm{S}_{j}} \text {. }
$$

(4) Let $m=\max \left\{\operatorname{dim}\left(\mathrm{S}_{i}\right)\right\}_{i \in \mathscr{I}}$. Then,

$$
\mathrm{X}_{\mathrm{reg}}=\bigcup_{i \in \mathscr{I} \mid \operatorname{dim} S_{i}=m} \mathrm{~S}_{i}
$$

is an open dense subset, called the regular part of the stratification.

(5) Let $\mathscr{I}^{\prime}$ be the subset of indices $i \in \mathscr{I}$ such that $\operatorname{dim}\left(\mathrm{S}_{i}\right)<m$. If $\mathscr{I}^{\prime}$ is not empty, then let $\mathscr{S}^{\prime}=\left\{\mathrm{S}_{i}\right\}_{i \in \mathscr{I}^{\prime}}$ and $\mathrm{X}^{\prime}=\cup_{i \in \mathscr{I}^{\prime}} \mathrm{S}_{i}$, equipped with the subset diffeology. Then $\mathscr{S}^{\prime}$ is a stratification of $\mathrm{X}^{\prime}$.

The space $\mathrm{X}$ is assumed to be Hausdorff, metrisable and connected for the D-topology. The subset $\mathrm{X}^{\prime}$ wich is equal to $\mathrm{X}-\mathrm{X}_{\text {reg }}$ is called the singular part of the stratification, and can be sometimes denoted by $\mathrm{X}_{\text {sing. }}$. The elements of $\mathscr{S}^{\prime}$ are called the singular strata. The first four axioms can be called the structure axioms and the fifth, the recursion axiom. For topological stratified spaces, the definition can be found in Pflaum survey [Pfl01]. By this recursion we get a filtration of diffeological subspaces:

$$
\mathrm{X}_{0} \subset \mathrm{X}_{1} \subset \cdots \subset \mathrm{X}_{\ell} \subset \cdots \subset \mathrm{X}_{k-1} \subset \mathrm{X}_{k} .
$$

where $\mathrm{X}_{k}=\mathrm{X}, \mathrm{X}_{k-1}=\mathrm{X}^{\prime}$ etc. Each term $\mathrm{X}_{\ell}$ of the filtration is a stratified space with the according subset $\mathscr{S}_{\ell}$ of strata, and the main strata has some dimension $n_{\ell}$. Each term being the singular part of the term above.

The next step consists in specializing the structure near the singular strata. We said that the stratification is locally fibered [GIZ18] — or the space is a locally fibered stratified spaces - if there exists a tube system $\left.\left\{\pi_{\mathrm{S}}: \mathrm{TS} \rightarrow \mathrm{S}\right)\right\}_{\mathrm{S} \in \mathscr{S}}$ such that:

(A) TS is an open neighborhood of S, called a tube over/around S.

(B) The map $\pi_{S}$ : TS $\rightarrow S$ is a smooth retraction which is a diffeological fibration.

(C) For all $x \in \mathrm{TS}^{\prime} \cap \mathrm{TS}^{\prime} \cap \pi_{\mathrm{S}^{\prime}}^{-1}(\mathrm{TS})$, one has $\pi_{\mathrm{S}}\left(\pi_{S^{\prime}}(x)\right)=\pi_{\mathrm{S}}(x)$.

The fiber $\mathrm{F}_{x}=\pi_{\mathrm{S}}^{-1}(x)$, with $x \in \mathrm{S}$, is a diffeological space itself which is stratified inherited by the ambient stratification. Its intersection with the stratum is reduced to one point: $\mathrm{F}_{x} \cap \mathrm{S}=\{x\}$. We call it the apex of the fiber.

\footnotetext{
${ }^{1}$ See $[\mathrm{PIZ13}, \mathbb{\$} 4.1]$ for manifolds as diffeologies.
} 

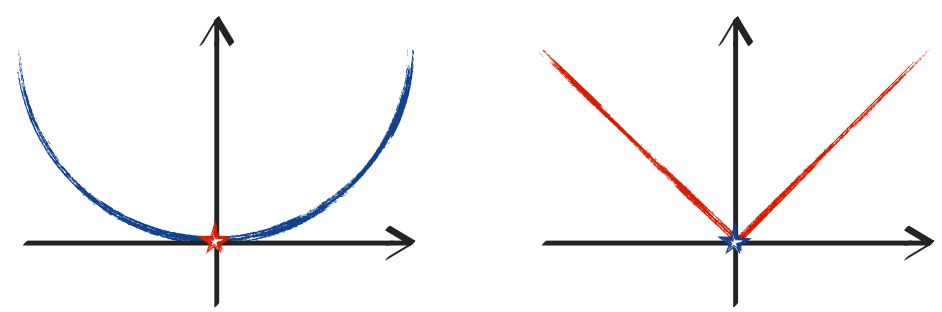

Figure 1. - A formal cone (left) and a geometric cone (right).

NOTE. - To be locally fibered is not a priori recursive. Do we need a natural recursion on the local fibration? This is a legitimate question, but we do not need this property for the case treated in this paper.

2. Diffeological Cones. Let $\Sigma$ be a diffeological space. We call a cone over $\Sigma$ the quotient space $\operatorname{Cone}(\Sigma)=\Sigma \times[0, \infty[/ \Sigma \times\{0\}$, equipped with the quotient diffeology. We recall that the quotient diffeology is the finest diffeology on the quotient that makes the projection smooth [PIZ13, $\$ 1.50]$. Consider now a diffeological space $\mathrm{F}$ with a smooth bijection $\varphi$ : $\operatorname{Cone}(\Sigma) \rightarrow \mathrm{F}$ such that $\varphi\lceil[\operatorname{Cone}(\Sigma)-\star]$ is a diffeomorphism onto its image, where $\star=\operatorname{class}(\Sigma \times\{0\})$ is the apex of the cone. Thus, without their apex, these spaces are equivalent to $\Sigma \times] 0, \infty[$. Then, $\mathrm{F}$ will be called a diffeological cone, or simply a cone, $\Sigma$ is called the base of the cone. The cone F differs from the cone over $\Sigma$ only by the germ of the diffeology at the apex.

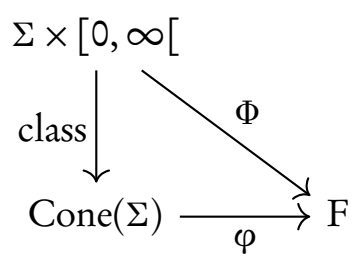

This consideration is necessary because diffeology discriminates between cones, as shows for example the positive cone in $\mathbf{R}^{3}$ defined by $x^{2}+y^{2}=z^{2} \& z \geq 0$, equipped with the subset diffeology, compared with the cone over $\mathrm{S}^{1}$, Cone $\left(\mathrm{S}^{1}\right)=$ $S^{1} \times\left[0, \infty\left[/ S^{1} \times\{0\}\right.\right.$ see $[G I Z 18, \mathbb{\$} 3]$.

Note. - An equivocal situation arises with this definition, related to the different types of stratified spaces [GIZ18, Introduction]. We have called geometric stratified spaces, the stratified spaces for which the strata are the (connected components of the) orbits of the pseudogroup of local diffeomorphisms. The formal stratified spaces are the other ones. The same situation happens with diffeological cones when we compare the two examples sketched in Fig. 1 above: the hemisphere (left cone) defined by $x^{2}+$ $y^{2}+(z-1)^{2}=1$ with $0 \leq z<1$, and the embedded cone (right cone) defined by $x^{2}+$ $y^{2}-z^{2}=0$, both equipped with the subset diffeology of $\mathbf{R}^{3}$. What differentiates these two cones is clearly the behavior of the apex $\star$. On the left cone it is interchangeable 
with any other point. Precisely, the pseudogroup of local diffeomorphisms does not distinguish the apex from the other points. On the other hand, the apex of the right cone is alone in its orbit. That observation leads us to distinguish at least two classes of cones:

DeFINITION We shall say that a cone is a geometric cone if its apex is alone in its orbit by the psendogroup of local diffeomorphisms. Otherwise, we shall say that it is a formal cone.

This remark has a real content, related to the idea about what is a cone. Intuitively, the apex of a cone should be distinguished. In diffeology it is the role of local diffeomorphisms to discriminate between points.

3. Differential Forms On Conelike Stratifed Diffeological spaces. We have introduced the general notion of locally fibered stratified space in [GIZ18]. They are diffeological spaces, together with a stratification, such that the space looks like a diffeological fiber bundle [PIZ13, $\$ 8.8,8.9]$ on the neighborhood of each stratum. This is a priori a two step generalization of the standard situation in the classical theory of stratified spaces, where the fiber is assumed to be conelike over a stratified base, as it is stated in [Sie72]. Hence, we have a diffeological intermediary to consider, the analogue of the usual conelike stratified spaces:

Definition - We call a Locally Conelike Diffeological Stratified Spaces any locally fibered stratified diffeological space with fibers some diffeological cones.

Note first that, in a locally conelike diffeological stratified space, the bases of the cones are stratified spaces as well. Secondly, the apex of the fibers is their intersection with the stratum.

Then, let $\alpha \in \Omega_{0}^{k}[\mathrm{X}]$ be a zero-perverse form defined on the regular part $\mathrm{X}_{\text {reg }}$ of a diffeological stratified space $\mathrm{X}$, and let $\nu$ the index function on zero-perverse forms, defined in [GIZ18, \$5].

Proposition - If $\mathrm{X}$ is a locally conelike diffeological stratified space, then there exists a (unique) differential form $\underline{\alpha} \in \Omega^{k}(\mathrm{X})$ such that $\alpha=\underline{\alpha}\left\lceil\mathrm{X}_{\mathrm{reg}}\right.$, if and only if $\nu_{\mathrm{S}}(\alpha)=1$ for all strata $\mathrm{S}$.

Note 1. Actually, the proposition applies to every stratum which is in the closure of the regular part. But since, by definition, $\mathrm{X}_{\text {reg }}$ is dense in $\mathrm{X}$ then the property applies for all strata.

Note 2. The proof of the proposition could be easily extended to the case of locally fibered diffeological stratified spaces with contractible fibers, maybe with some condition on the retraction with respect to the connected components of the regular part. But that would be a minor generalisation.

Proof. If $\nu_{\mathrm{S}}(\alpha)=1$ for all $\mathrm{S} \in \mathscr{S}$, then there exists a (unique) differential form $\underline{\alpha} \in$ $\Omega^{k}(\mathrm{X})$ such that $\alpha=\underline{\alpha}\left\lceil\mathrm{X}_{\text {reg. }}\right.$. That has been proven in a general context in [GIZ18]. 
Let then $\underline{\alpha} \in \Omega^{k}(\mathrm{X})$. For all strata $\mathrm{S}$, let $\pi_{\mathrm{S}}: \mathrm{TS} \rightarrow \mathrm{S}$ be the retraction of the tube around a singular stratum $\mathrm{S}$, which is by hypothesis a local fibration with fiber a cone F with base $\Sigma$. Recall that $S \subset$ TS and for all $x \in S, \pi_{S}(x)=x$.

Let $\alpha=\underline{\alpha}\left\lceil\mathrm{X}_{\text {reg }}\right.$, and assume that $\alpha$ is zero-perverse with respect to these local structures. To follow the construction of the index $\nu$ we consider the pullback of the tube TS by the projection of the universal covering pr: $\tilde{S} \rightarrow S$, even if it is not completely necessary, since our considerations will be local. Let us denote by TS $\hat{S}$ the pullback $\operatorname{pr}^{*}(\mathrm{TS})$, that is,

$$
\mathrm{TS}=\left\{(\tilde{x}, y) \in \tilde{\mathrm{S}} \times \mathrm{TS} \mid \operatorname{pr}(\tilde{x})=\pi_{\mathrm{S}}(y)\right\}
$$

This pullback is actually a tube around the embbeding $\hat{S}$ of the universal covering $\tilde{S}$ by the smooth section $\sigma: \tilde{x} \rightarrow(\tilde{x}, \operatorname{pr}(\tilde{x}))$.

$$
\hat{\mathrm{S}}=\{(\tilde{x}, \operatorname{pr}(\tilde{x})) \mid \tilde{x} \in \tilde{S}\} \subset \mathrm{T} \hat{S},
$$

which justifies the notation. Indeed, since $\operatorname{pr}(\tilde{x}) \in \mathrm{S}, \operatorname{pr}(\tilde{x})=\pi_{\mathrm{S}}(\operatorname{pr}(\tilde{x}))$ which is the condition for $(\tilde{x}, \operatorname{pr}(\tilde{x}))$ to belong to $\mathrm{TS}$, and obviously $\operatorname{pr}_{1} \circ \sigma=1_{\tilde{S}}$. Now,

$$
\pi_{\hat{\mathrm{S}}}:(\tilde{x}, y) \rightarrow\left(\tilde{x}, \pi_{\mathrm{S}}(y)\right),
$$

is a retraction from $\mathrm{TS}$ to $\hat{\mathrm{S}}$. The tube $\mathrm{TS}$ is also a fibration with the same fiber $\mathrm{F}$, it is the deployment of the tube TS along the embedded universal covering $\hat{S}$.

Hence, $\mathrm{TS}$ is everywhere locally diffeomorphic to a product $\mathscr{O} \times \mathrm{F}$, where $\mathscr{O} \subset \hat{\mathrm{S}}$ is some open subset, and the projection $\pi_{\hat{S}}:(\tilde{x}, y) \rightarrow\left(\tilde{x}, \pi_{S}(y)\right)$ locally equivalent to the first projection $\mathrm{pr}_{1}: \mathscr{O} \times \mathrm{F} \rightarrow \mathscr{O}$. That means that there exists a local diffeomorphism $\psi$, defined on $\mathscr{O} \times \mathrm{F}$, into $\mathrm{TS}$ such that $\pi_{\hat{\mathrm{S}}} \circ \psi=\mathrm{pr}_{1}$, We choose always $\mathscr{O}$ to be connected and simply connected. Now, the regular part of $\pi_{\hat{\mathrm{S}}}^{-1}(\mathscr{O})$ is the image by $\psi$ of the regular part of the product $\mathscr{O} \times \mathrm{F}$, that is, $\mathscr{O} \times \mathrm{F}_{\text {reg. }}$. But $\mathrm{F}_{\text {reg }}$ is naturally identified to $] 0, \infty\left[\times \Sigma_{\text {reg }}\right.$, by construction. Therefore we have the following chain of square commutative diagrams.

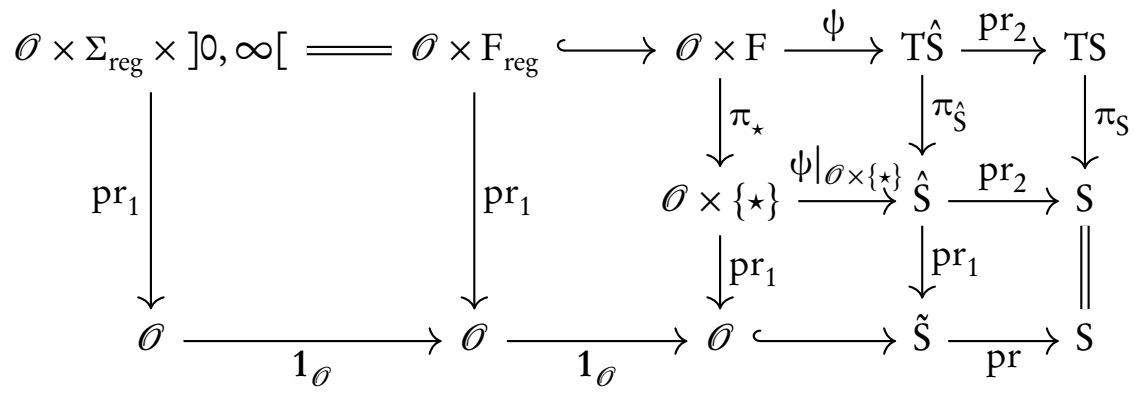

We need to make a remark here about the arrow $\left.\psi\right|_{\mathscr{O} \times\{\star\}}: \mathscr{O} \times\{\star\} \rightarrow \hat{\mathrm{S}}$ that associates $(\tilde{x}, \star)$ with $(\tilde{x}, x=\operatorname{pr}(\tilde{x}))$. For geometric cones, this arrow exists by construction since the apex is alone in its orbit by the pseudogroup of local diffeomorphisms. 


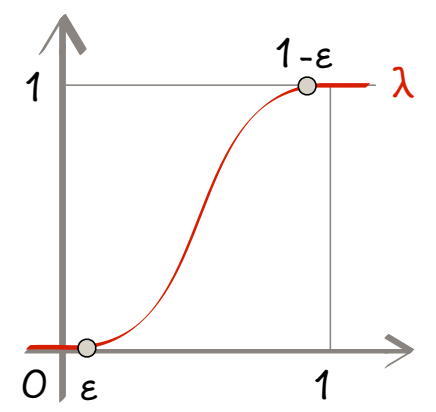

FIGURE 2. - The smashing function $\lambda$.

For formal cones only, the local triviality should be understood in the category of stratified spaces. Let us now introduce the following notations:

$$
\left.\hat{\alpha}=\operatorname{pr}_{2}^{*}(\underline{\alpha}) \in \Omega^{k}(\mathrm{~T} \hat{S}), \quad \text { and } \quad \tilde{\alpha}=\hat{\alpha}\right\rceil \mathrm{TS}_{\text {reg }}=\operatorname{pr}_{2}^{*}(\alpha) \in \Omega^{k}\left(\mathrm{TS}_{\text {reg }}\right) \text {. }
$$

Next, we decompose $\mathrm{F}_{\text {reg }}$ in terms of connected components and have:

$$
\left.\mathscr{O} \times \mathrm{F}_{\text {reg }}=\mathscr{O} \times \coprod_{a} \mathrm{~F}_{\text {reg }}^{a}=\mathscr{O} \times \coprod_{a} \Sigma_{\text {reg }}^{a} \times\right] 0, \infty\left[\text { with } a \in \pi_{0}\left(\Sigma_{\text {reg }}\right) .\right.
$$

And we get a two-squares diagram summarizing the situation we will deal with:

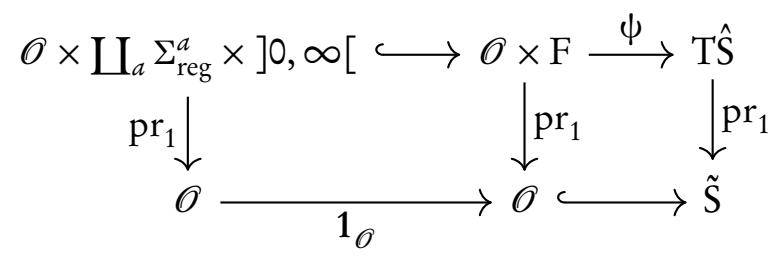

Now, consider a $n$-plot $\mathrm{P}$ in $\tilde{\mathrm{S}}$ defined on some open set $\mathrm{U}$, and $r_{0} \in \mathrm{U}$. There exists an open neighborhood $\mathrm{V} \subset \mathrm{U}$ of $r_{0}$ such that $\mathrm{Q}=\mathrm{P} \uparrow \mathrm{V}$ takes its values in some trivialization open set $\mathscr{O}$, which we assume to be connected and simply connected. Now let $\left.y \in \mathrm{F}_{\text {reg }}=\coprod_{a} \Sigma_{\text {reg }}^{a} \times\right] 0, \infty\left[\right.$, then $y=\Phi(z, t)$ for some $z \in \Sigma_{\text {reg }}^{a}$ and $t \in] 0, \infty[$, where $\Phi$ has been introduced in (art. 2). Consider then the (stationary) smooth path

$$
\gamma: s \mapsto \Phi(z, \lambda(s) t)
$$

where $\lambda$ is the smashing function described by Fig. 2 .

Now:

(1) For all $s \in]-\infty, \varepsilon], \gamma(s)=\star$ is the apex of $\mathrm{F}$.

(2) For all $s \in] \varepsilon, \infty\left[, \gamma(s) \in \mathrm{F}_{\text {reg. }}^{a}\right.$.

(3) In particular $\gamma(0)=\star$ and $\gamma(1)=y$. 
Next, let $\mathrm{Q} \times \gamma$ be the plot $(r, s) \mapsto(\mathrm{Q}(r), \gamma(s))$, into $\mathscr{O} \times \mathrm{F}$, when $(r, s)$ runs over $\mathrm{V} \times \mathbf{R}$. Let us fix $\left(u_{i}\right)_{i=1}^{k}$ with $u_{i} \in \mathbf{R}^{n}$, and $\left(\tau_{i}\right)_{i=1}^{k}$ with $\tau_{i} \in \mathbf{R}$. We consider then the real smooth function defined on $\mathrm{V} \times \mathbf{R}$,

$$
f(r, s)=\hat{\alpha}(\psi \circ(\mathrm{Q} \times \gamma))_{(r, s)}\left(u_{i}, \tau_{i}\right)_{i=1}^{k}
$$

Indeed: $\psi \circ(\mathrm{Q} \times \gamma)$ is a plot in $\mathscr{O} \times \mathrm{Y}, \psi$ is a local diffeomorphism into $\mathrm{TS}, \hat{\alpha}$ is a $k$-form on $\mathrm{TS}$, and the $\left(u_{i}, \tau_{i}\right)_{i=1}^{k}$ have been fixed.

(1) Consider firstly $s \in]-\infty, \varepsilon\left[\right.$, thus $\gamma(s)=\star$. Let us denote $\tilde{x}_{r}=\mathrm{Q}(r)$, then $\psi(\mathrm{Q}(r), \gamma(s))=\psi\left(\tilde{x}_{r}, \star\right)=\left(\tilde{x}_{r}, x_{r}\right)$, with $x_{r}=\operatorname{pr}\left(\tilde{x}_{r}\right)$. Hence, we have:

$$
\begin{aligned}
f(r, s) & =\hat{\alpha}\left((r, s) \mapsto \psi\left(\tilde{x}_{r}, \star\right)\right)_{(r, s)}\left(u_{i}, \tau_{i}\right)_{i=1}^{k} \\
f(r, s) & =\hat{\alpha}\left((r, s) \mapsto\left(\tilde{x}_{r}, x_{r}\right)\right)_{(r, s)}\left(u_{i}, \tau_{i}\right)_{i=1}^{k} \\
& =\hat{\alpha}\left((r, s) \mapsto r \mapsto\left(\tilde{x}_{r}, x_{r}\right)\right)_{(r, s)}\left(u_{i}, \tau_{i}\right)_{i=1}^{k} \\
& =\operatorname{pr}_{1}^{*}\left(\hat{\alpha}\left(r \mapsto\left(\tilde{x}_{r}, x_{r}\right)\right)\right)_{(r, s)}\left(u_{i}, \tau_{i}\right)_{i=1}^{k} \\
& =\hat{\alpha}\left(r \mapsto\left(\tilde{x}_{r}, x_{r}\right)\right)_{r}\left(u_{i}\right)_{i=1}^{k} \\
& =\underline{\alpha}\left(r \mapsto x_{r}\right)_{r}\left(u_{i}\right)_{i=1}^{k}, \text { because } \hat{\alpha}=\operatorname{pr}_{2}^{*}(\underline{\alpha}) \\
& =\underline{\alpha}(\operatorname{proQ})_{r}\left(u_{i}\right)_{i=1}^{k} .
\end{aligned}
$$

Therefore

$$
f \uparrow \mathrm{V} \times]-\infty, \varepsilon\left[=\operatorname{pr}^{*}(\underline{\alpha})(\mathrm{Q})_{r}\left(u_{i}\right)_{i=1}^{k} .\right.
$$

(2) Consider secondly $s \in] \varepsilon,+\infty\left[\right.$. Then, $\gamma(s) \in \mathrm{F}_{\text {reg }}^{a}, \mathrm{Q} \times \gamma$ is a plot in $\mathscr{O} \times \mathrm{F}_{\text {reg }}^{a}$ and $\psi \circ(\mathrm{Q} \times \gamma)$ is a plot of $\{\mathrm{TS}\}_{a}$, the connected component of $\mathrm{TS}=\mathrm{pr}^{*}(\mathrm{TS})$ associated with the component $\left.\mathrm{F}_{\text {reg }}^{a}=\Sigma_{\text {reg }}^{a} \times\right] 0, \infty[$, see [GIZ18, $\$ 5$, Step 1]. Hence, according

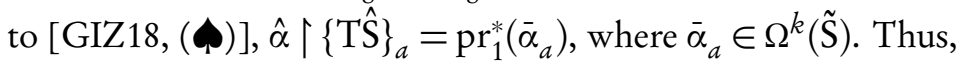

$$
\begin{aligned}
f(r, s) & =\hat{\alpha}\left((r, s) \mapsto \psi\left(\tilde{x}_{r}, \gamma(s)\right)\right)_{(r, s)}\left(u_{i}, \tau_{i}\right)_{i=1}^{k} \\
& =\operatorname{pr}_{1}^{*}\left(\bar{\alpha}_{a}\right)\left((r, s) \mapsto \psi\left(\tilde{x}_{r}, \gamma(s)\right)\right)_{(r, s)}\left(u_{i}, \tau_{i}\right)_{i=1}^{k} \\
& =\bar{\alpha}_{a}\left(\operatorname{pr}_{1} \circ\left[(r, s) \mapsto \psi\left(\tilde{x}_{r}, \gamma(s)\right)\right]\right)_{(r, s)}\left(u_{i}, \tau_{i}\right)_{i=1}^{k} \\
& =\bar{\alpha}_{a}\left((r, s) \mapsto \operatorname{pr}_{1} \circ \psi\left(\tilde{x}_{r}, \gamma(s)\right)\right)_{(r, s)}\left(u_{i}, \tau_{i}\right)_{i=1}^{k} \\
& =\bar{\alpha}_{a}\left((r, s) \mapsto \tilde{x}_{r}\right)_{(r, s)}\left(u_{i}, \tau_{i}\right)_{i=1}^{k}, \text { since } \operatorname{pr}_{1} \circ \psi=\operatorname{pr}_{1} \\
& =\bar{\alpha}_{a}\left(r \mapsto \tilde{x}_{r}\right)_{r}\left(u_{i}\right)_{i=1}^{k} .
\end{aligned}
$$

Therefore,

$$
f \uparrow \mathrm{V} \times] \varepsilon, \infty\left[=\bar{\alpha}_{a}(\mathrm{Q})_{r}\left(u_{i}\right)_{i=1}^{k} .\right.
$$

(3) In conclusion - For all $r \in \mathscr{O} f_{r}: s \mapsto f(r, s)$ is a smooth function defined on R. On the interval $]-\infty, \varepsilon\left[, f_{r}\right.$ is constant and equal to $\operatorname{pr}^{*}(\underline{\alpha})(\mathrm{Q})_{r}\left(u_{i}\right)_{i=1}^{k}$, and on $] \varepsilon,-\infty\left[, f_{r}\right.$ is constant and equal to $\bar{\alpha}_{a}(\mathrm{Q})_{r}\left(u_{i}\right)_{i=1}^{k}$. Since $f_{r}$ is smooth, then 
continuous, these two constants must be equal. Thus, since that is true locally for any $r$ and any vectors $u_{i}$ in $\mathbf{R}^{n}$, we get

$$
\bar{\alpha}_{a}=\operatorname{pr}^{*}(\underline{\alpha} \mid S)
$$

for all indices $a$. That is, $\nu(S)=1$. Now, that applies to every stratum which is in the closure of $\mathrm{X}_{\mathrm{reg}}$. Since by definition $\mathrm{X}_{\mathrm{reg}}$ is dense in $\mathrm{X}, \nu(\mathrm{S})=1$ for all strata.

\section{REFERENCES}

[GIZ18] Serap Gürer and Patrick Iglesias-Zemmour. Differential Forms On Stratified Spaces. To appear in the Bulletin of the Australian Mathematical Society https://doi.org/10.1017/S0004972718000436

[PIZ13] Patrick Iglesias-Zemmour Diffeology. Mathematical Surveys and Monographs. The American Mathematical Society, vol. 185, USA R.I. 2013.

[Klo07] Benoît Kloeckner. Quelques Notions d'Espaces Stratifiés. Séminaire de Théorie Spectrale et Géométrie. Vol. 26, pp. 13-28, Grenoble 2007-08.

[Pfl01] Markus Pflaum. Analytic and geometric study of stratified spaces. Lecture notes in Mathematics, vol.1768, Springer verlag, 2001.

[Sie72] Larry Siebenmann. Deformation of Homeomorphisms on Stratified Sets. Comment. Math. Helv. vol. 47, pp. 123-163, 1972.

Serap GÜrer - Galatasaray University, Ortaköy, ÇıraĞan CD. No:36, 34349 BeşikTaş / İstanbul, TURKEY.

E-mail address: sgurer@gsu.edu.tr

Patrick Iglesias-Zemmour - Aix Marseille Univ, CNRS, Centrale Marseille, I2M, Marseille, France $-\&-$ Einstein Institute of Mathematics Edmond J. Safra Campus, The Hebrew UniVersity of Jerusalem Givat Ram. Jerusalem, 9190401, Israel.

E-mail address: piz@math.huji.ac.il 